\title{
Design and Simulation of a Flow Mobility Scheme Based on Proxy Mobile IPv6
}

\author{
Hyon-Young Choi*, Sung-Gi Min*, Youn-Hee Han* and Rajeev Koodli ${ }^{\star \star *}$
}

\begin{abstract}
Proxy Mobile IPv6 (PMIPv6) is a network-based mobility support protocol and it does not require Mobile Nodes (MNs) to be involved in the mobility support signaling. In the case when multiple interfaces are active in an $\mathrm{MN}$ simultaneously, each data flow can be dynamically allocated to and redirected between different access networks to adapt to the dynamically changing network status and to balance the workload. Such a flow redistribution control is called "flow mobility". In the existing PMIPv6-based flow mobility support, although the MN's logical interface can solve the well-known problems of flow mobility in a heterogeneous network, some missing procedures, such as an $\mathrm{MN}$-derived flow handover, make PMIPv6-based flow mobility incomplete. In this paper, an enhanced flow mobility support is proposed for actualizing the flow mobility support in PMIPv6. The proposed scheme is also based on the MN's logical interface, which hides the physical interfaces from the network layer and above. As new functional modules, the flow interface manager is placed at the MN's logical interface and the flow binding manager in the Local Mobility Anchor (LMA) is paired with the MN's flow interface manager. They manage the flow bindings, and select the proper access technology to send packets. In this paper, we provide the complete flow mobility procedures which begin with the following three different triggering cases: the MN's new connection/disconnection, the LMA's decision, and the MN's request. Simulation using the ns-3 network simulator is performed to verify the proposed procedures and we show the network throughput variation caused by the network offload using the proposed procedures.
\end{abstract}

Keywords—Flow Mobility, Proxy Mobile IPv6

\section{INTRODUCTION}

The future network will be comprised of various heterogeneous access networks in order to realize global accessibility and diverse networking resources will converge together to satisfy user and application requirements. Equally, with the rapid growth in the number of mobile nodes (MNs) such as cellular phones, smartphones, and laptop computers, the demand for seamless

\footnotetext{
※ This paper extends the conference version that was presented at the second workshop on mobility modeling and performance evaluation in 2011 (MoMoPe2011) [16]. This research was supported by the IT R\&D support program of KCA (KCA-10913-05004)

Manuscript received February 29, 2012; first revision June 29, 2012; accepted September 12, 2012.

Corresponding Author: Sung-Gi Min

* Department of Computer and Radio Communications Engineering, Korea University, Seoul, Korea (\{neongas, sgmin\}@korea.ac.kr)

** School of Computer Science and Engineering, Korea University of Technology and Education, CheonAn, Korea (yhhan@koreatech.ac.kr)

*** Cisco Systems, 3650 Cisco Way, San Jose, CA 95134, USA (rkoodli@cisco.com)
} 
mobility services such as Voice over IP (VoIP) is becoming one of the most important issue in mobility management. Moreover, multiple interfaces have become recently available to user devices for the accessing of heterogeneous multiple access networks. A solution of such a multiple access case is the vertical handover, (i.e., the handover between different access networks). In the case when multiple interfaces can be active in a host, the vertical handover provides an effective mechanism to seamlessly switch the network connection when necessary.

When an $\mathrm{MN}$ transfers an on-going session from one access network to another, the IP address will change. In order to allow a Corresponding Node $(\mathrm{CN})$ that the $\mathrm{MN}$ is communicating with to find the location of the MN correctly and to allow the session to continue, an IP mobility management should be used. The network-based IP mobility management protocol called Proxy Mobile IPv6 (PMIPv6) [1] was actively standardized by the IETF netext (network-based mobility extensions) working group, and has begun to attract a lot of attention from the telecommunication community as well as from the Internet community. Unlike the host-based IP mobility management protocols such as Mobile IPv6 (MIPv6) [2] and Dual-Stack Mobile IPv6 [3], PMIPv6 is expected to expedite the real deployment of IP mobility management.

Multiple access network connectivity can generally be simultaneously available. For example, when a smartphone tha is equipped with both $3 \mathrm{G}$ and WLAN interfaces enters into the overlapping areas of the two access networks. In the case when multiple interfaces can be active in an MN simultaneously, each data flow (i.e., a sequence of packets matching a flow description) can be dynamically allocated to and redirected between different access networks to adapt to the dynamically changing network status and balance the workload. More specifically, the benefits of the simultaneous connection to multiple networks include the following items:

- A system can deliver each service flow via the access network that is most suitable and efficient for it, and thus provide a wide range of Quality of Service (QoS)/Quality of Experience (QoE) to a user.

- An application can have several connections and allow each traffic to use the access network that is most suitable to its characteristics.

- A specific type of access network must be kept available to provide some services with the required QoS/QoE. In this case, one interface should not be masked by another one due to the presence of a mobility management mechanism.

Traffic flows can be dynamically distributed to diverse access networks by allowing a host to use multiple interfaces simultaneously and thus, the future network can provide novel applications with new styles of networking support. In this case, the flow level for traffic control is needed in addition to host level mobility management.

In this paper, we introduce a novel PMIPv6-based architecture and scheme to support multiconnectivity management and flow mobility. Our design to support flow mobility is based on the logical interface that is in the MN. Logical interface hides all of the physical interfaces from the network layer and above. In this paper, we introduce two major components-theflow interface manager and the flow binding manager-to manage flow mobility. The flow interface manager is placed at the MN's logical interface layer and the flow binding manager in the LMA's network layer is paired with it. They manage the flow bindings that are used to select proper access technology through which packets are sent. The flow mobility procedure is 
divided into three cases, 1) new connection from the MN, 2) the LMA's decision, and 3) the MN's decision and LMA's consent.

We implemented our PMIPv6-based flow mobility scheme using the ns-3 network simulator [17]. The ns-3 is a new simulator that is intended to eventually replace the aging ns-2. Ns-3 has a good development momentum, and is believed to have a better core architecture and to be better suited to receive community contributions. It should be especially noted that ns-3 fully supports multiple interfaces in a node whereas ns-2 cannot support them originally. By using these ns-3 features, we implemented the multi-interfaced MNs and the flow mobility procedure in the proposed architecture. We also provided flow mobility test scenarios and ran them on our simulation system. From the simulation results, we verified that the proposed architecture and scheme satisfy the flow mobility requirements well.

The rest of this paper is organized as follows: Section 2 introduces the PMIPv6's feature and basic operation and other approaches for supporting flow mobility. Section 3 describes the proposed architecture, logical interface layer and flow handover procedures. Section 4 gives a simulation configuration, scenario and results. In Section 5, the conclusions of this paper are provided.

\section{PMIPV6 AND ITS EXTENSIONS}

The PMIPv6's features and basic operation are described clearly in [4]. In this paper, we briefly describe them here in order to understand our proposals without difficulty.

The foundation of PMIPv6 is based on MIPv6 in the sense that it extends MIPv6 signaling and reuses many concepts such as the Home Agent (HA) function. However, PMIPv6 is designed to provide network-based mobility management support to an $\mathrm{MN}$ in a topologically localized domain. Therefore, an MN is exempted from participation in any mobility-related signaling, and the proxy mobility agent in the serving network performs mobility-related signaling on behalf of the MN. Once an MN enters its PMIPv6 domain and performs access authentication, the serving network ensures that the $\mathrm{MN}$ is always on its home network and that it can obtain the Home Address (HoA) on any access network. That is, the serving network assigns a unique Home Network Prefix (HNP) to each MN, and conceptually this prefix always follows the MN wherever it moves within a PMIPv6 domain. From the perspective of the MN, the entire PMIPv6 domain appears as its home network. Accordingly, it is needless (or impossible) to configure the Care-of-Address (CoA) in the MN.

As shown in Fig. 1, the new principal functional entities of PMIPv6 are the Mobile Access Gateway (MAG) and the Local Mobility Anchor (LMA). The MAG typically runs on the AR. The main role of the MAG is to detect the MN's movements and initiate mobility-related signaling with the MN's LMA on behalf of the MN. In addition, the MAG establishes a tunnel with the LMA for enabling the MN to use an address from its HNP and emulates the MN's home network on the access network for each MN. On the other hand, the LMA is similar to the HA in MIPv6. However, it has the additional capabilities that are required to support PMIPv6. The main role of the LMA is to maintain reachability to the MN's address while it moves around within a PMIPv6 domain, and it includes a binding cache entry for each currently registered MN. The binding cache entry maintained at the LMA is more extended than that of the HA in MIPv6 as it has some additional fields such as the MN-Identifier, the MN's HNP, a flag indicating a proxy regis- 


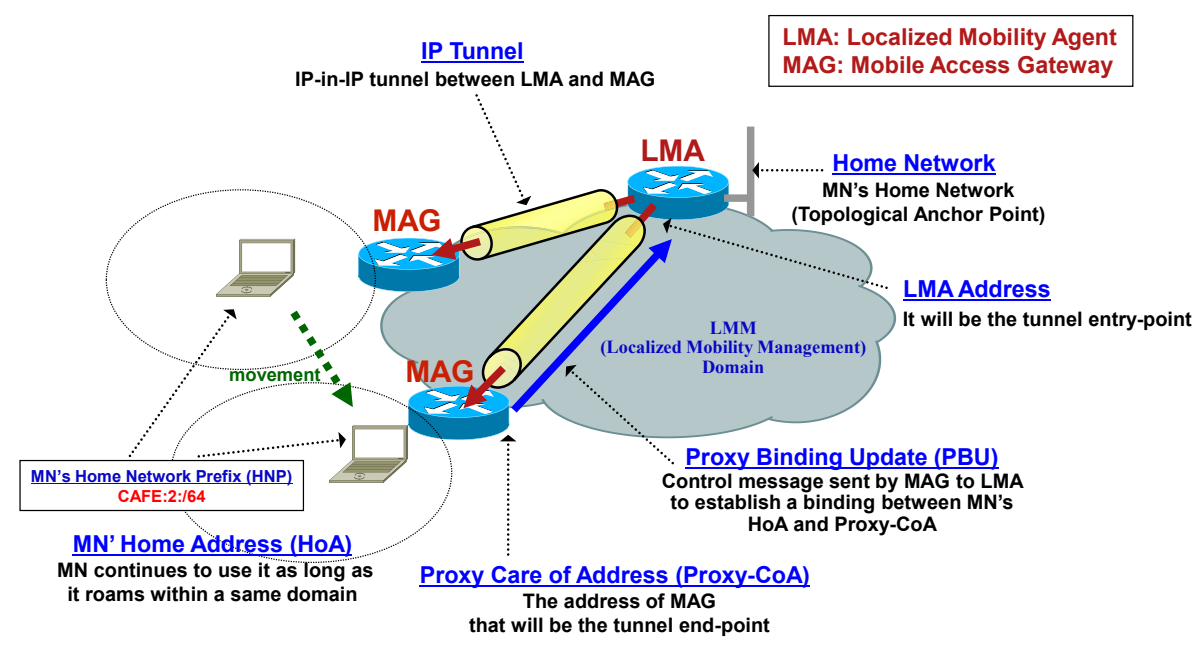

Fig. 1. Overview of PMIPv6 [1]

tration, and the interface identifier of the bidirectional tunnel between the LMA and MAG. Such information associates an $\mathrm{MN}$ with its serving MAG, and enables the relationship between the MAG and LMA to be maintained.

When an MN first attaches to an access network connected to the MAG, an authentication procedure is performed using an MN's identity (i.e., MN-Identifier) via the deployed access security protocols. After successful access authentication, the MAG sends a Proxy Binding Update (PBU) message including the MN-Identifier to the LMA on behalf of the MN. Once the LMA receives the $\mathrm{PBU}$ message, it checks the policy store to ensure that the sender is authorized to send the PBU message. If the sender is a trusted MAG, the LMA accepts the PBU message and sends a Proxy Binding Acknowledgment (PBA) message including the MN's HNP, to the MAG. It also sets up a route for the MN's HNP through the tunnel to the MAG. Once the MAG receives the PBA message from the LMA, it starts to emulate the MN's home network on the access network, and it sends a Router Advertisement (RA) message containing the MN's HNP to the MN. It is noted that since PMIPv6 only supports the per-MN prefix model and not the shared-prefix model, a unique HNP is assigned to each MN. Therefore, the MN always configures its unique home address while it moves within a PMIPv6 domain.

There are several primitive solutions for supporting flow mobility in PMIPv6 [5-7]. These approaches try to support flow mobility without MN's involvement due to the PMIPv6's inherent policy. However, performing an inter-technology handover only in the network side without involvement from the MN has many practical issues [8]. One of these major issues is that the session cannot be sustained because the addresses configured at the multiple interfaces are created based on a different interface identifier and thus all of them are not identical. Therefore, the support of a PMIPv6-based inter-technology handover means that inevitably MNs should be modified. Moreover, in order to move a flow from an interface to another and to sustain the traffic session, the IP address constituting the session must be identical across the two interfaces. Due to these reasons, logical interface supports [10-12] are proposed to support multi-homing, vertical handover, and flow mobility in network-based mobility management. 
If a logical interface as well as physical interface is installed in an $\mathrm{MN}$, the network layer always exchanges data only with the logical interface. That is, the network layer and above layers think that there is only one interface even if the MN has many physical interfaces. Using the logical interface, the MN must have the dispatch module to decide which physical interface is chosen for sending data traffic to. On the other hand, all packets that arrive at physical interfaces are forwarded to the logical interface first, and then, they are delivered to the network layer.

A scheme that is proposed in an IETF Internet draft [13] is a representative solution to support flow mobility in PMIPv6 ${ }^{1}$. It also assumes that $\mathrm{MN}$ has a logical interface as well as multiple physical interfaces. However, the solution given in [13] has some limitations. First, it does not support the MN-initiated flow mobility at all. Although PMIPv6 has a network-based approach and thus flow handover from an interface to another should be performed based on the network's decision, a user controlling the MN may want to redirect one flow of traffic from the currently served interface to another. Any flow mobility solution would not be complete if it does not support the $\mathrm{MN}$-initiate flow mobility. In this paper, we provide network-based flow mobility schemes that are similar to the proposals in [13] and also present a new procedure where an $\mathrm{MN}$ can try to redirect one flow of traffic from one interface to another. Second, current works does not have a detailed definition of the flow and fails to describe the flow mobility management performed in the LMA and the MAG. In this paper, we provide concrete procedures of flow mobility management as well as the flow definition. Third, the trigger that initiates a flow mobility operation is not exactly specified in [13], where we explain the three triggering cases. In addition, we verify and evaluate our proposals through intensive simulation by using ns-3.

\section{PMIPv6-BASEd FLoW MoBILITY SCHEME}

\subsection{System Architecture and Updated Stack Structures of MN and LMA}

In this paper, we consider a PMIPv6-based mobility domain as described in Fig. 2. The domain refers to the network where the mobility management of MNs is handled using the PMIPv6 protocol as defined in [1]. We assume that each MAG has an interface connected with only one access technology. That is, two different access technologies (e.g., 3G and WLAN) do not have a connection with an MAG. We also assume that each MN has one or more network interfaces.

Fig. 2 also depicts the MN and LMA's stack architecture that has been modified to support flow mobility. The MN's stack has the logical interface layer placed between the network layer and the data link layer. It consists of the logical interface and the flow interface manager. All packets from the lower and upper layers are always passed through the logical interface layer. The network layer and upper layers only see the logical interface as their network interface. As explained in Section 2, the utilization of a logical interface can solve the many problems that can happen when host-level vertical handover or flow handover should be controlled in a networkbased mobility management scheme. The flow interface manager stores the flow interface bindings and selects the proper physical interface for an outbound flow. On the other hand, the LMA also manages the flow binding in its flow binding manager. For an inbound flow, the flow binding manager selects the proper tunnel for the flow to get to an MAG. The flow binding and flow

1 One of this paper's authors take participate in writing the Internet draft. 


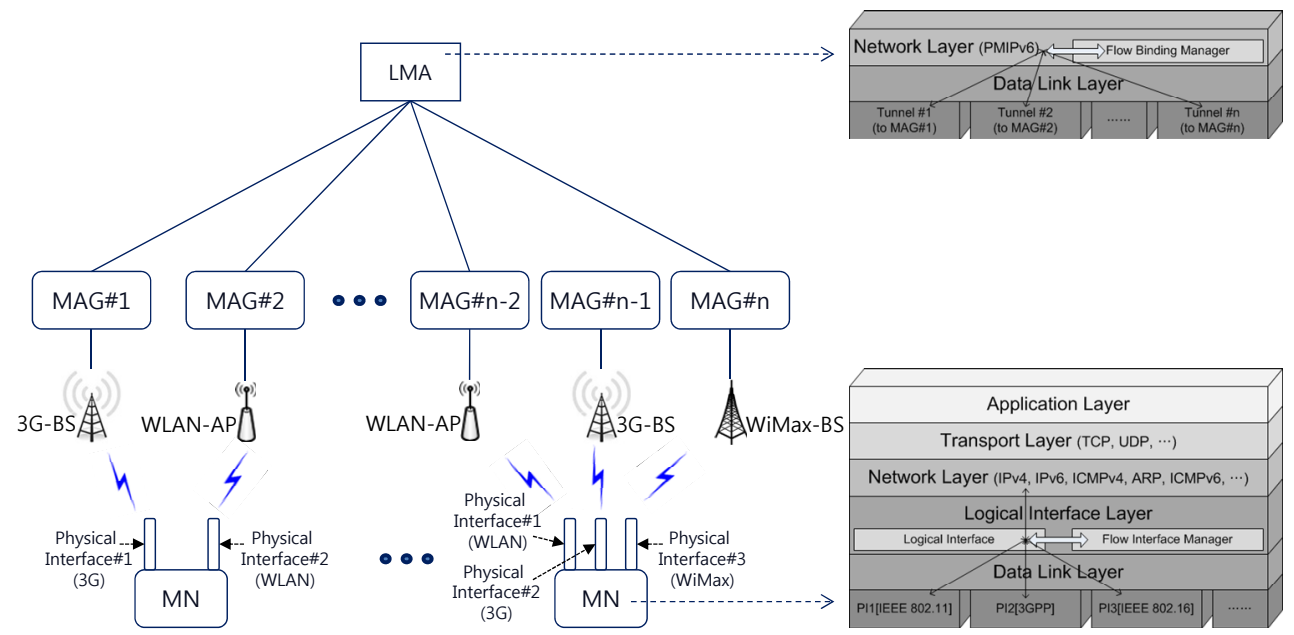

Fig. 2. System Architecture and Updated Stack Structures of MN and LMA

interface binding will be explained below.

\subsection{Flow, Flow Selector and Flow Binding Information}

To manage and process flow handover, the definitions of the flow and flow selector are needed in advance. In this paper, a flow is defined as a series of packets matching a particular flow description. To define a flow description, the various parameters mentioned for defining a flow in [9] can be used. The significant and frequently used parameters from among these are the 5tuple parameters, which consist of the source address, destination address, source port, destination port, and protocol. These 5-tuple parameters are used for a flow description.

Since a flow is represented with 5-tuple information, it thus contains direction information and a flow can be categorized into two different types-inbound flow and outbound flow (see Fig. 3). Inbound flow is sent from a $\mathrm{CN}$ and is finally received at the MN through the LMA and MAGs, while outbound flow is sent from the MN to a CN through MAGs and the LMA. The LMA should determine the MAG through which a particular inbound flow should be delivered. Whereas, MN should determine its physical interface (and thus the MAG) though which a particular outbound flow should be sent. If the descriptions of a flow and another flow are $<i p \_x$, $i p \_y$, port_x, port_y, proto col_z $>$ and $\left\langle i p \_y\right.$, ip_x, port_y, port_x, protocol_z $>$, respectively, we call the two flows symmetrically congruent. In this paper, we define a bidirectional session as a symmetrically congruent pair of flows. If a session is unidirectional, a flow can be a session. From the perspective of the MN, we require that two symmetrically congruent flows in a session must be open at the same Access Technology Type (ATT) (i.e., the same physical interface).

The flow selector is the information used for flow filtering to decide which MAG the packets of a particular flow are forwarded to. The definition of a flow selector is similar to that of flow. The difference is that the 5-tuple of flow selector can contain a wildcard symbol $(*$, asterisk) to match any value. For example, any TCP flow sent from a web server for which the address is $3 f f e: 34 a b:: 11 f$ can be filtered by the flow selector $<3 f f e: 34 a b:: 11 f$, *, 80, *, TCP>.

In our proposal, the binding information between the flow selector and the priority list of ATTs are managed as flow binding in the LMA. On the other hand, the MN maintains the flow 


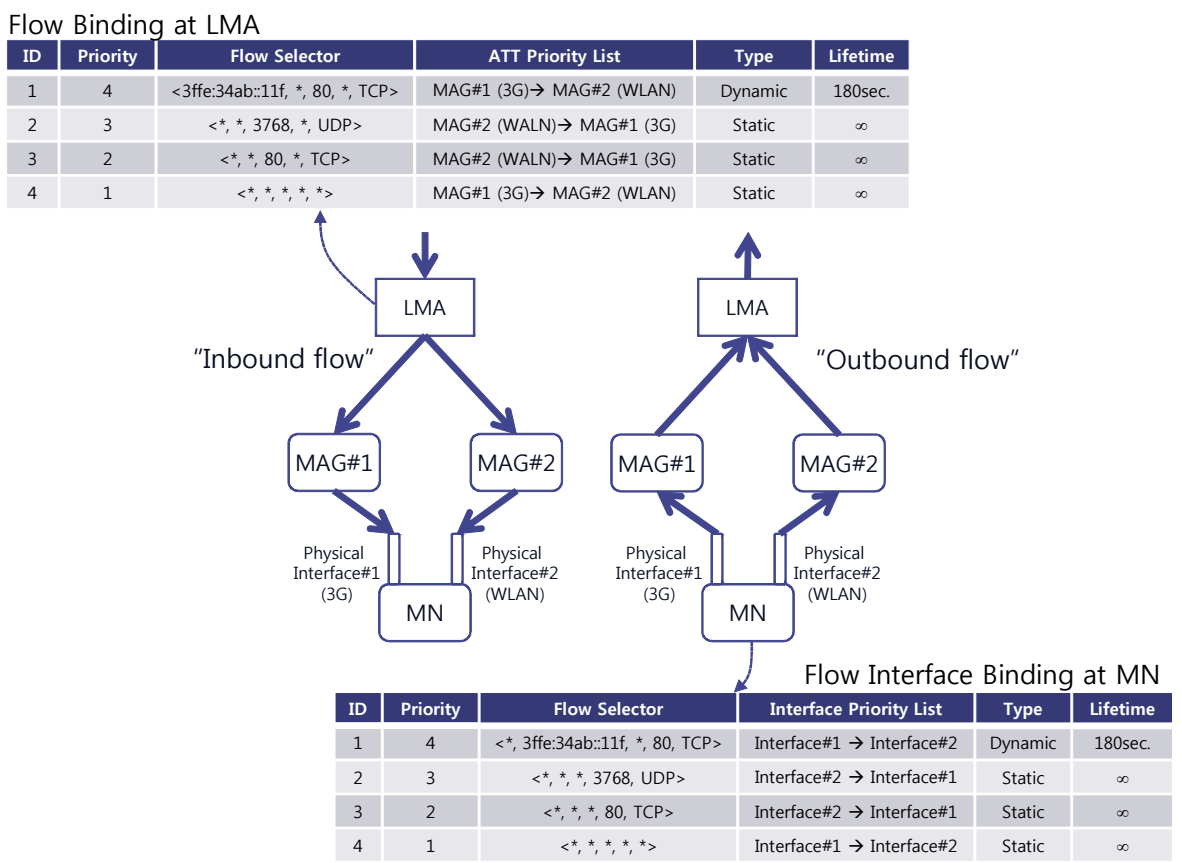

Fig. 3. Two Types of Flow Binding Information: Flow Binding in the LMA and Flow Interface Binding in the MN

interface binding where the binding information between the flow selector and the priority list of its physical interfaces are stored. Each flow binding information in the LMA contains the flow ID, priority, the flow selector, the priority list of ATTs, the binding type, and lifetime fields. Each entry is identified by a flow ID. The filtering order of each entry is based on the priority field (high values represent high priority). If an inbound flow in the LMA is filtered by a flow selector in the flow binding information, the ATT with the highest priority is selected from among the associated list of ATTs and thus the corresponding tunnel is used for sending the inbound flow. The flow interface binding in the MN is similar to the flow binding in the LMA, but it has the priority list of physical interfaces instead of a list of ATTs. The list is used for selecting the outgoing interface for the filtered outbound flow.

It should be noted that two symmetrically congruent flow selectors in the LMA's flow binding and MN's flow interface binding have the same priority list in terms of ATTs. In this paper, we assume that the flow interface binding information of LMA and MNs comes from policy profile such as the Authentication, Authorization, and Accounting (AAA) server. When an MN connects to the network, it performs an authentication process with the AAA server and gets the flow interface binding information from the policy profile. The LMA also can get the information during the initial binding update process. We call these types of binding information static information which is stored in the type field of the LMA's flow binding and in the MN's flow interface binding information.

In addition, LMA and MNs can manage dynamic flow interface binding information. If the MN wants to redirect one flow of traffic to the other interface, it sends a request for flow mobility to the LMA. The request includes the flow binding information that is being requested to be 
moved by the MN. When the LMA receives flow binding information that is different from what the LMA gets in its static information, it first determines whether or not this type of flow mobility is acceptable and inserts a new dynamic flow binding into its flow binding information if it is acceptable. We think that the network (that is, the LMA) should control the flow mobility since our design should follow the PMIPv6's policy of network-based mobility control. This is because the LMA determines if an MN's flow mobility request is acceptable prior to performing dynamic binding insertion. Similarly, when receiving an inbound flow from an access technology that is different from what the MN gets in its static information, the MN just inserts a new dynamic flow interface binding into its flow interface binding information. A dynamic type of binding entry in both the LMA and MN has a finite lifetime and it is managed as a kind of softstate.

\subsection{Flow Mobility Procedure}

In most literature, flow-level handover has been not studied much. So, we need to first explain when flow-level handover can occur. The flow-level handover can be initiated by the following three triggering cases:

1) A new connection/disconnection: when an MN makes a connection (or disconnection) with a network through a physical interface, the flow-level redistribution, which is based on the flow binding information in both the LMA and MN, can be performed.

2) LMA's decision at any time: the LMA can get link conditions such as congestion, the number of serving nodes, or the number of flows, since the LMA is the topological anchor for all of the traffic in the PMIPv6 domain. Therefore, the LMA can start the flow handover by its own decision to offload a network.

3) MN's request and LMA's approval: an MN may want to move a particular flow from the served interface to another interface. In the PMIPv6 domain, which follows the networkbased mobility management, the network (i.e., LMA) should grant the MN's request for flow handover. Hence, the MN must send a request to the LMA for flow handover, and it can move the requested flow after receiving the LMA's approval.

When a new physical interface is activated, if all of the flows are being delivered through higher preferred interfaces than the new interface, there is no flow handover. Otherwise, some of the flows will move to the new interface. According to [1], when an MN connects to a PMIPv6 domain through multiple interfaces for simultaneous access, the LMA should allocate different HNP for each of the attached interfaces and then each mobility session associated with an HNP should be managed under a separate binding cache entry. However, in order to support flow mobility a new interface can be allocated the same HNP as the one used in the existing interfaces if some of existing the flows need to be moved to the new interface.

Fig. 4 shows the flow handover procedure that is caused by a new connection. Prior to the new connection, there are three flows-flows $\mathrm{X}, \mathrm{Y}$, and $\mathrm{Z}$ over interface 1 (if1). As different HNPs have been assigned to each flow, the RA delivers all of the HNPs of the flows. If it is assumed that the new interface 2 (if2) is the higher preferred interface for flow Y, then flow $\mathrm{Y}$ will be moved into the new interface when interface 2 is connected and activated. Because flow handover is expected for the new interface, the LMA assigns HNP2 to the new interface during 


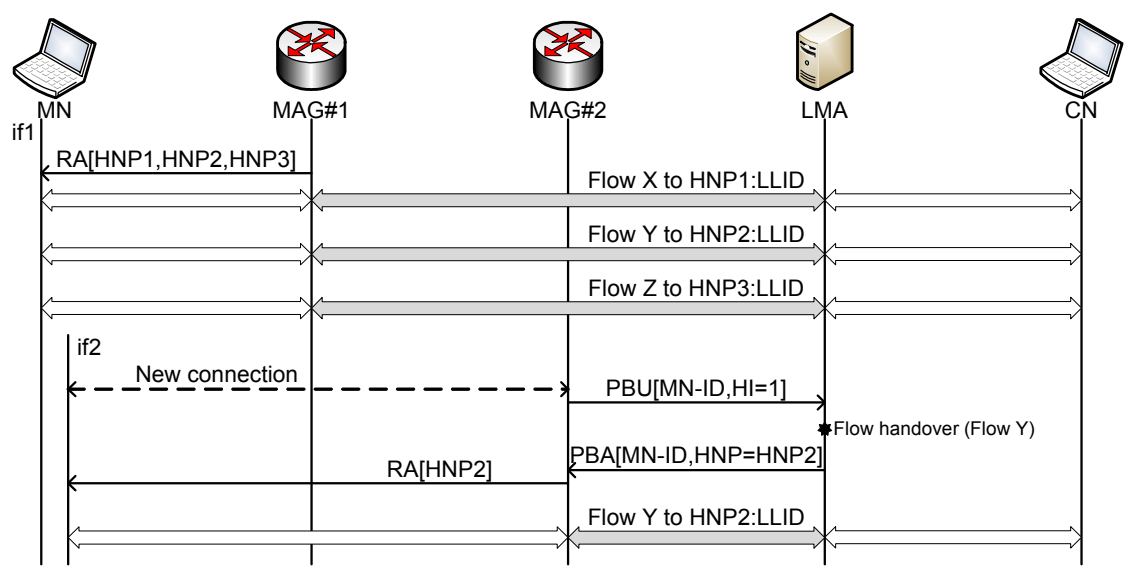

Fig. 4. Flow Handover Procedure by New Connection

the binding update process.

For the second case, when the LMA decides to redirect one flow to the other at any time, it has two possible actions based on whether the HNP of the target flow has been assigned to the target interface. If the target interface has the HNP of the target flow, flow handover is performed immediately. Otherwise, it needs an additional procedure to assign the HNP of the target flow to the target interface. In this paper, the HNP Update Request (HUR) and HNP Update Acknowledge (HUA) [14] are utilized for this process before flow handover.

Fig. 5 shows the flow handover for the second case. The status of the initial flows is that flows $\mathrm{X}$ and $\mathrm{Z}$ are using interface 1 and flow $\mathrm{Y}$ is using interface 2 for communicating between the $\mathrm{CN}$ and the $\mathrm{MN}$.

Since it is assumed that flow $\mathrm{Y}$ has been moved to interface 2, both MAG\#1 and MAG\#2

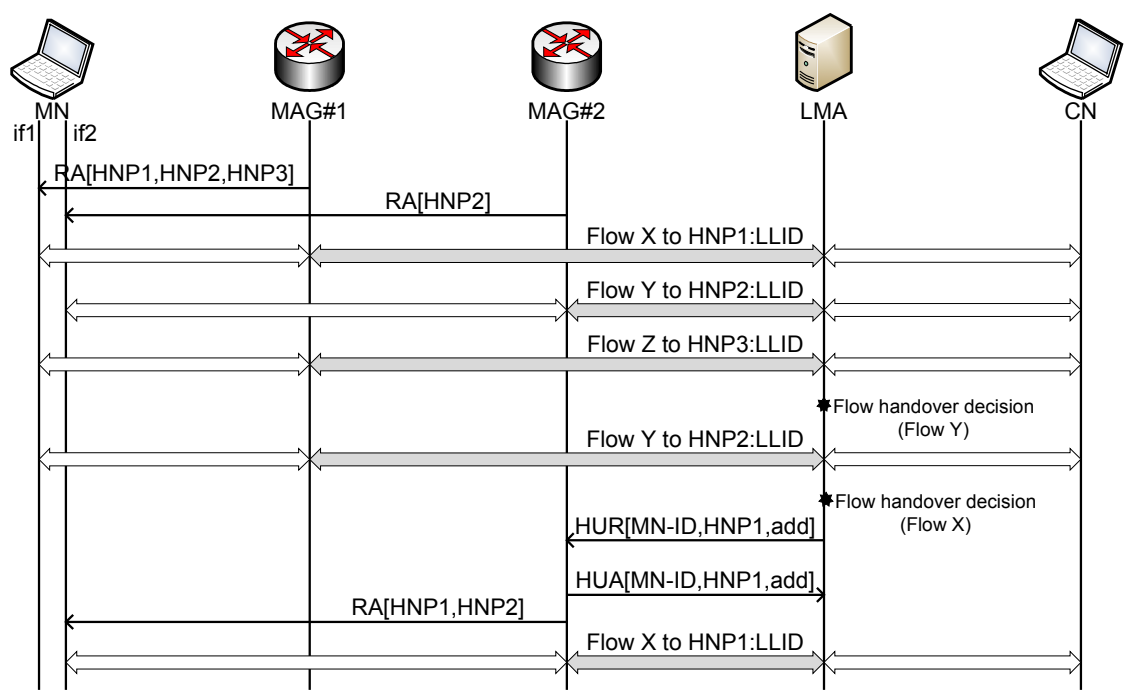

Fig. 5. Flow Handover Procedure by LMA's Decision at any Time 


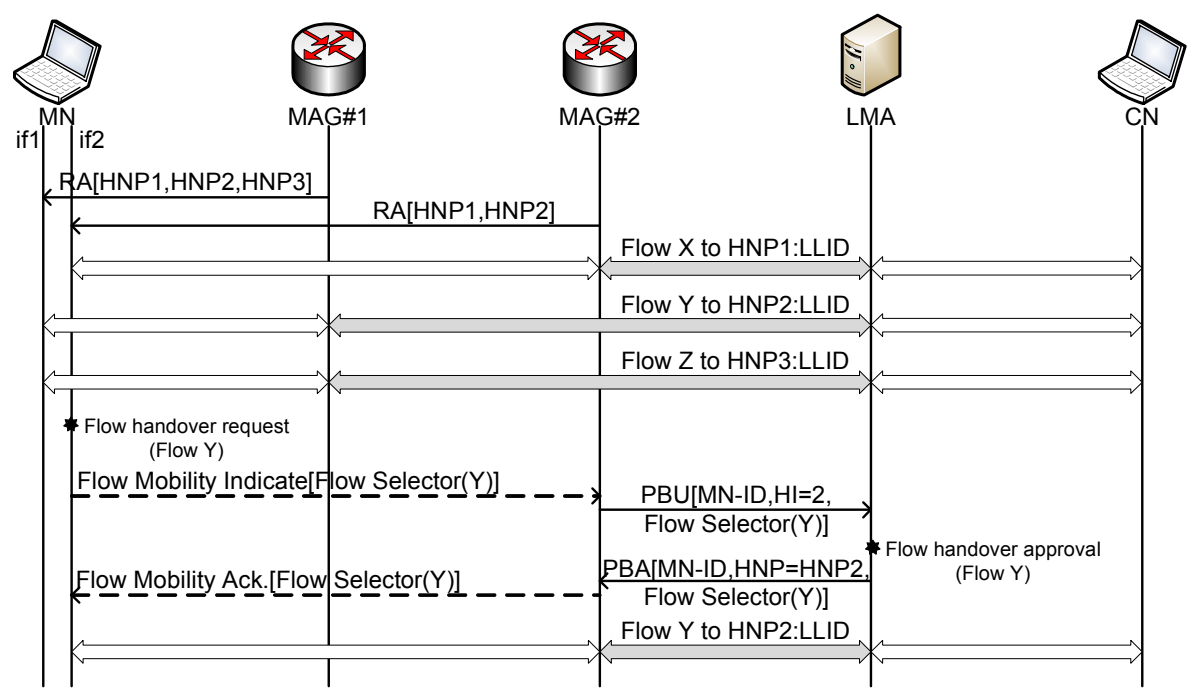

Fig. 6. Flow Handover Procedure by MN's request and LMA's approval

send an RA message with the HNP2. If the LMA decides to move flow Y to interface 1 again, it can be easily moved to interface 1 because MAG\#1 already knows the HNP2 for flow Y. However, if it is decided that flow $\mathrm{X}$ should move to interface 2, the LMA assigns the HNP2 to MAG\#2 using HUR/HUA messages before flow handover. As a result, the RA message contains both HNP1 and HNP2.

When the MN wants to move some flow to another interface (target interface), it should notify the MAG, to which the target interface is connected to, of this request. New messages, Flow Mobility Indicate and Flow Mobility Acknowledge, are defined for the flow mobility request that has been delivered from the MN to the MAG. It is assumed that these two messages should be delivered as a network layer signal. For example, like PBU and PBA, they can be implemented by using new mobility headers $[1,2]$. They have only one option, which is called the flow selector option. This option contains the flow selector which is used for filtering the flow that is requested to redirect. The PBU and PBA messages containing the flow selector option can be used for transferring the flow binding information between the MAG and LMA.

Fig. 6 depicts the MN-derived flow handover procedure. As initial status, flow $\mathrm{X}$ is using if 2 and flows $\mathrm{Y}$ and $\mathrm{Z}$ are using if1. When the $\mathrm{MN}$ wants to move flow $\mathrm{Y}$ from if2 to if1, it sends a Flow Mobility Indicate message with the flow selector of flow Y through the target interface. The MAG transfers the MN's request to the LMA using the PBU message with the flow selector. If the LMA approves the request, it sends the PBA message back to the MAG, and the MAG sends the Flow Mobility Acknowledge message to the MN. After receiving flow handover approval from the LMA, the MN moves flow Y to if1.

\section{SimULATION AND RESULTS}

The ns-3 network simulator $[15,17]$ was used to perform a simulation of flow mobility support. 
Fig. 7 illustrates the network topology with the three physical interfaces of WLAN, WiMax(WiBro), and 3G(PPP). Many CNs are connected to the border router, and can access the PMIPv6 domain network by passing through the LMA. The LMA manages three MAGs. The wired link speed is $100 \mathrm{Mbps}$, and the link delay is $0.01 \mathrm{~ms}$ for all of the links. The link speeds of WLAN, WiMax, and 3G are $54 \mathrm{Mbps}, 75 \mathrm{Mbps}$, and $1 \mathrm{Mbps}$, respectively. The interface queue size is 100 packets for a wired link, 400 packets and 5 seconds of lifetime for WLAN, 1,024 packets including control packets for WiMax, and 100 packets for PPP. In order to not change the MN's default gateway after the MN receives the RA, all of the MAG's MAC addresses on the edge network interface are unified to "00:00:AA:BB:CC:DD." Hence, the same link-local addresses for MAGs are shown in the figure. The MN has a logical interface with the Link-ID: "00:00:11:22:33:44."

There are five UDP traffic flows from the CNs to the MN as shown in Table 1. All of the traffic uses HNP1 as the destination address, which is assigned to the first activated interface. To distinguish each UDP flow, the source and destination ports are allocated different numbers. The start times of UDP flows are varied based on the scenario to validate the proposed flow handover cases. The UDP1, UDP2 and UDP3 flows are related to the flow handover, while the UDP0.1 and UDP0.2 flows are used as background traffic.

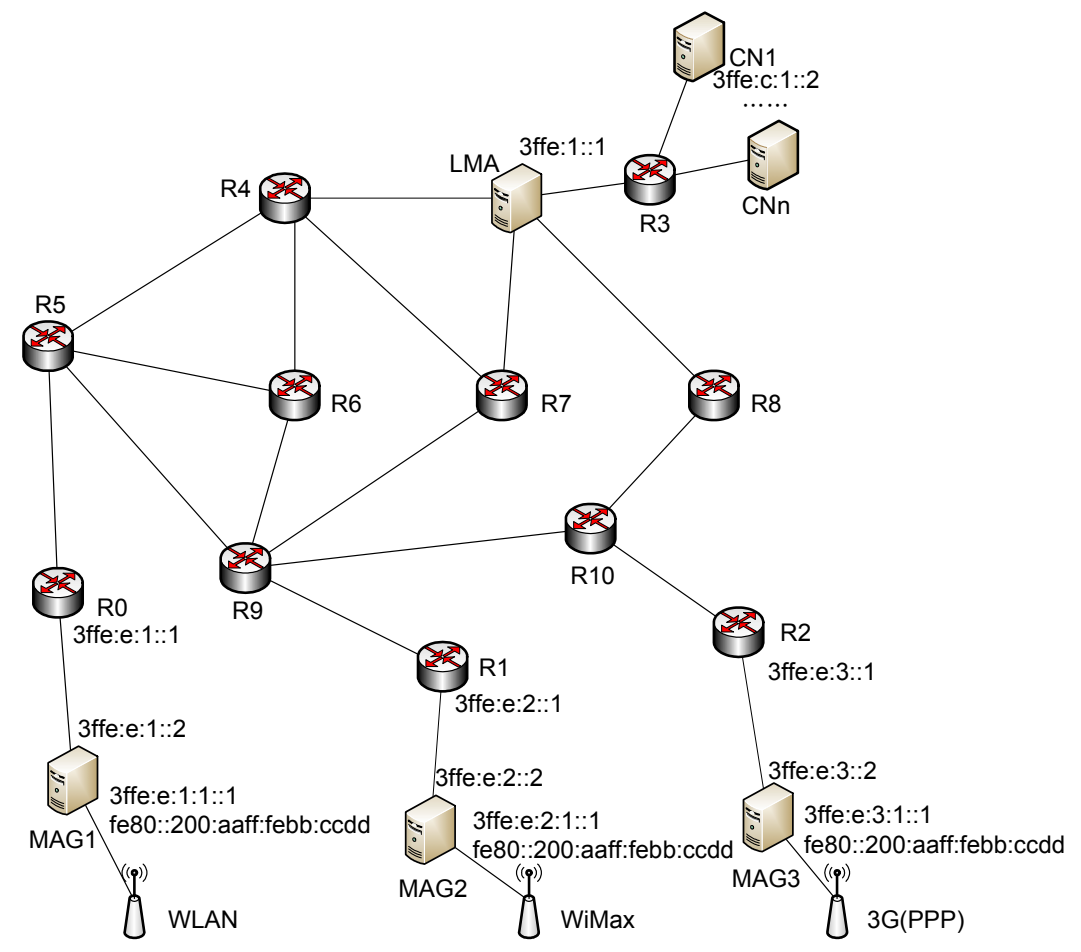

fe80::200:11ff:fe22:3344

(Virtual Interface)

Fig. 7. Simulation Topology 
Table 1. Traffic Description

\begin{tabular}{c|c|c|c|c|c|c}
\hline Flow & Src. & Dest. & Proto. & Rate & Start & Stop \\
\hline UDP1 & CN:56 & MN:10 & UDP & $7 \mathrm{Mbps}$ & $2.0 \mathrm{~s}$ & $25.0 \mathrm{~s}$ \\
\hline UDP2 & CN:57 & MN:20 & UDP & $3 \mathrm{Mbps}$ & $2.0 \mathrm{~s}$ & $25.0 \mathrm{~s}$ \\
\hline UDP3 & $\mathrm{CN}: 58$ & $\mathrm{MN}: 30$ & UDP & $0.8 \mathrm{Mbps}$ & $12.0 \mathrm{~s}$ & $25.0 \mathrm{~s}$ \\
\hline UDP0.1 & $\mathrm{CN}: 59$ & $\mathrm{MN}: 40$ & UDP & $15 \mathrm{Mbps}$ & $2.0 \mathrm{~s}$ & $25.0 \mathrm{~s}$ \\
\hline UDP0.2 & $\mathrm{CN}: 60$ & $\mathrm{MN}: 50$ & UDP & $10 \mathrm{Mbps}$ & $14.0 \mathrm{~s}$ & $25.0 \mathrm{~s}$ \\
\hline
\end{tabular}

The Initial Flow Binding List and Initial Flow Interface List are shown in Table 2 and Table 3, respectively. The fields of the Binding ID and Interface ID only contain the priority list among access technology types because the activated interface ID and the matching binding ID cannot be determined before the beginning of the simulation. When an interface is activated or the PMIPv6 binding update process is completed, the proper binding ID or interface index will be filled to the field of the matched access technology type.

According to the lists, UDP2 is matched with Flow ID 2, UDP3 is matched with Flow ID 3 , and the other UDPs are matched with Flow ID 1. Hence, the highest priority among interfaces is WiMax for UDP2, 3G for UDP3, and WLAN for the others.

Table 4 shows the simulation scenario. This simulation scenario is set up to check the validity of the proposed procedure. The total simulation time is 25 seconds. It is divided into three phases. The first phase is the flow mobility procedure by new connection, the second phase is carried out by LMA's decision, and the third phase is done by MN's request and LMA's approval.

There are several detailed events for each phase and the reactions for each event are easily expected. When UDP1, UDP2, and UDP0.1 start to be sent at 2.0 seconds, they are delivered to the MN through MAG1 (WLAN) because WLAN is the only interface that is activated at that time. Since UDP2 has the highest priority for WiMax, when WiMax is activated at 5.0 seconds, it performs a flow handover from WLAN to WiMax. It can be expected that the MN's WiMax interface gets the same HNP as the MN's WLAN interface because the LMA knows that UDP2 will perform the flow handover when the WiMax interface is activated. The flow mobility for UDP1 and UDP2 does not happen when $3 \mathrm{G}$ is activated at 10.0 seconds. The MN's $3 \mathrm{G}$ interface gets new HNP which is different from the HNPs which are already allocated to the MN's WLAN and WiMax interfaces because there is no influenced flow for the activated 3G interface. When

Table 2. Initial Flow Binding List in the LMA

\begin{tabular}{c|c|c|c}
\hline Flow ID & Priority & Flow Selector & ATT Priority List \\
\hline 3 & 15 & $(*, *, *, 30, \mathrm{UDP})$ & $3 \mathrm{G}(-) \rightarrow \mathrm{WM}(-) \rightarrow \mathrm{WL}(-)$ \\
\hline 2 & 15 & $(*, *, *, 20, \mathrm{UDP})$ & $\mathrm{WM}(-) \rightarrow 3 \mathrm{G}(-) \rightarrow \mathrm{WL}(-)$ \\
\hline 1 & 10 & $(*, *, *, *, \mathrm{UDP})$ & $\mathrm{WL}(-) \rightarrow 3 \mathrm{G}(-) \rightarrow \mathrm{WM}(-)$ \\
\hline
\end{tabular}

Table 3. Initial Flow Interface List in the MN

\begin{tabular}{c|c|c|c}
\hline Flow ID & Priority & Flow Selector & ATT Priority List \\
\hline 3 & 15 & $(*, *, 30, *, \mathrm{UDP})$ & $3 \mathrm{G}(-) \rightarrow \mathrm{WM}(-) \rightarrow \mathrm{WL}(-)$ \\
\hline 2 & 15 & $(*, *, 20, *, \mathrm{UDP})$ & $\mathrm{WM}(-) \rightarrow 3 \mathrm{G}(-) \rightarrow \mathrm{WL}(-)$ \\
\hline 1 & 10 & $(*, *, *, *, \mathrm{UDP})$ & $\mathrm{WL}(-) \rightarrow 3 \mathrm{G}(-) \rightarrow \mathrm{WM}(-)$ \\
\hline
\end{tabular}


Table 4. Simulation Scenario

\begin{tabular}{c|c|l}
\hline Phase & Time $(\mathrm{s})$ & \multicolumn{1}{c}{ Event } \\
\hline \multirow{2}{*}{$\begin{array}{c}\text { Phase1 } \\
(0.0 \mathrm{~s}-10.0 \mathrm{~s})\end{array}$} & 0.0 & - Simulation starts \\
\cline { 2 - 3 } & 0.0 & - WLAN is activated \\
\cline { 2 - 3 } & 2.0 & - UDP1, UDP2 and UDP0.1 start to send \\
\hline \multirow{4}{*}{$\begin{array}{c}\text { Phase2 } \\
(10.0 \mathrm{~s}-20.0 \mathrm{~s})\end{array}$} & 5.0 & - WiMax is activated \\
\cline { 2 - 3 } & 10.0 & - 3G(PPP) is activated \\
\cline { 2 - 3 } & 12.0 & - UDP3 starts to send \\
\hline $\begin{array}{c}\text { Phase3 } \\
(20.0 \mathrm{~s}-25.0 \mathrm{~s})\end{array}$ & 14.0 & - UDP0.2 starts to send \\
\hline \multirow{2}{*}{-} & 20.0 & - LMA moves UDP1 to WiMax \\
\hline
\end{tabular}

UDP3 starts to be sent at 12.0 seconds, it must be sent to the MN's 3G interface which is the highest priority for UDP3. At 14.0 seconds, UDP0.2 starts to be sent via the MN's WLAN interface. This traffic causes the network congestion on the WLAN access network and the sending rate of UDP1 becomes low. Hence, the LMA itself enforces the flow handover of UDP1 to the MN's WiMax interface by inserting a new dynamic entry at 15.0 seconds. For a certain reason, the MN requests the flow handover of UDP3 from the MN's WLAN interface at 20.0 seconds.

Fig. 8 depicts the throughput of the flows for the first phase in the MN. After the activation of

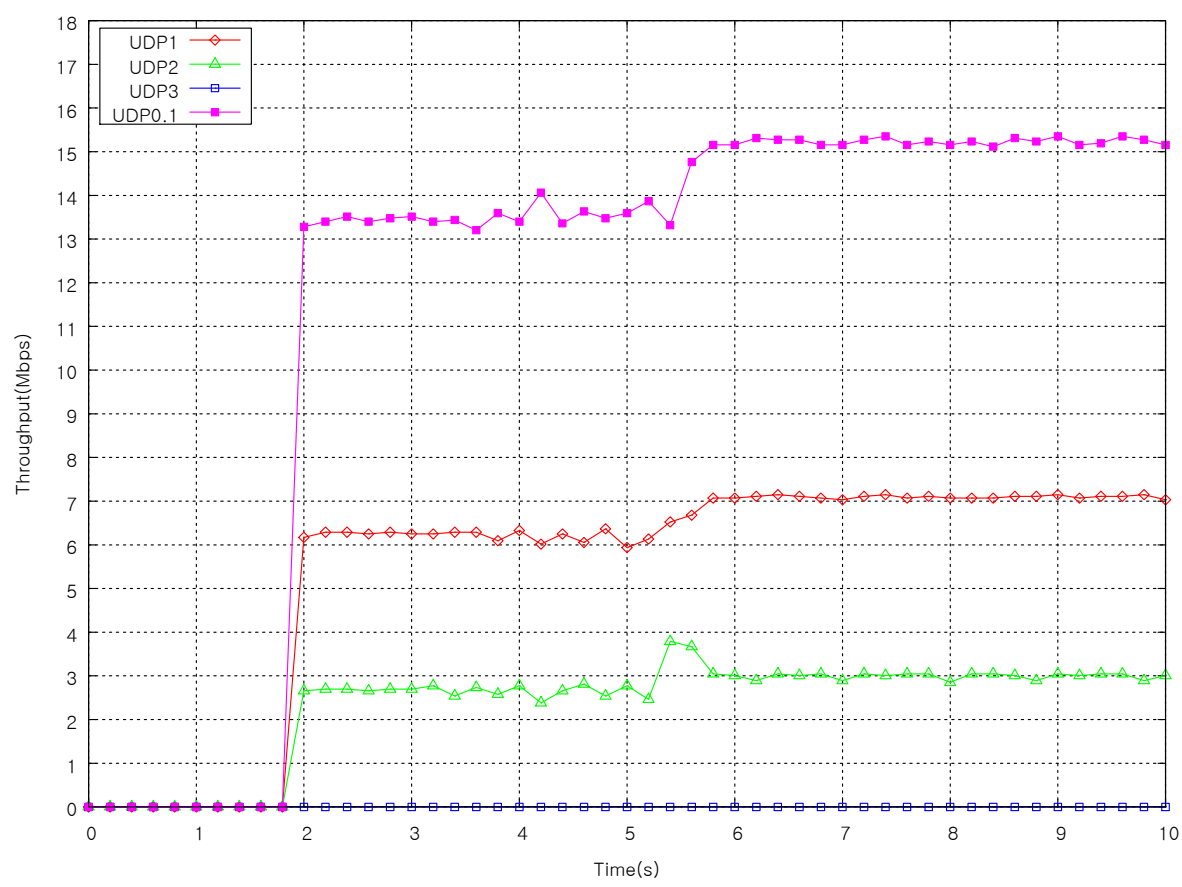

Fig. 8. UDP Throughput Trace of Phase 1 at the MN 
MN's WLAN interface at the beginning of the simulation, UDP1, UDP2, and UDP0.1 start to be sent via WLAN at 2.0 seconds. Since the bandwidth of WLAN is $54 \mathrm{Mbps}$, this traffic cannot be delivered at their full rate. When the WiMax's interface is activated at 5.0 seconds, the UDP2 flow is moved from the MN's WLAN interface to the MN's WiMax one because WiMax has higher priority than WLAN. It can be noticed that the throughput of UDP2 increases just after the flow handover and it is caused by the interface queue of the WLAN access point. When UDP flows are delivered by only using WLAN, the bandwidth of the wireless link is smaller than the arriving rate. Hence, the packets are queued in the interface queue of the WLAN access point. When the flow handover occurs, UDP2 packets in the interface queue are still sent to MN while UDP2 packets are delivered to the MN through WiMax. After this period, the UDP2 flow is delivered with the full serving rate and the throughput of both the UDP1 and UDP0.1 flows also increases.

The received packet sequence in the MN around the period of the UDP2's flow handover is shown in Fig. 9. The UDP1 flow is delivered with its serving rate continuously due to no flow handover in the first phase. After the flow handover of UDP2 at approximately 5.5 seconds, both interfaces are used for the UDP2 flow delivery. In this period, the throughput of UDP2 increases more than its serving rate and the $\mathrm{MN}$ receives packets with mixed sequences.

Fig. 10 depicts the throughput of the UDP flows for the second and the third phases. In the second phase, the UDP3 flow is sent at 12.0 seconds and the throughput of UDP1 and UDP0.1 flows that are delivered through WLAN decrease from 14.0 seconds due to the start of the UDP0.2 flow delivery. After the flow handover of the UDP1 flow to the MAG2 based on the LMA's decision at 15.0 seconds, the UDP1 flow is delivered with its full rate as well as the

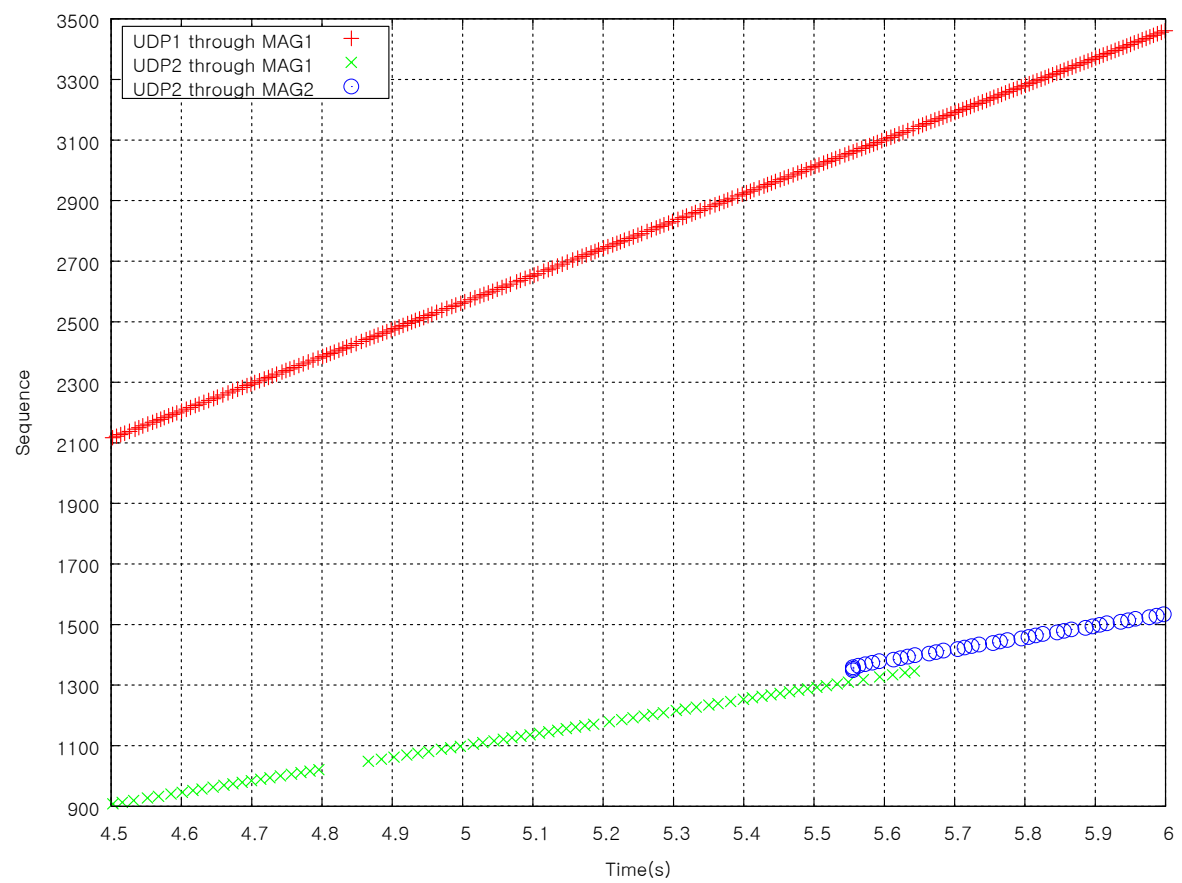

Fig. 9. Packet Sequence at the MN around the period of UDP2's flow handover 


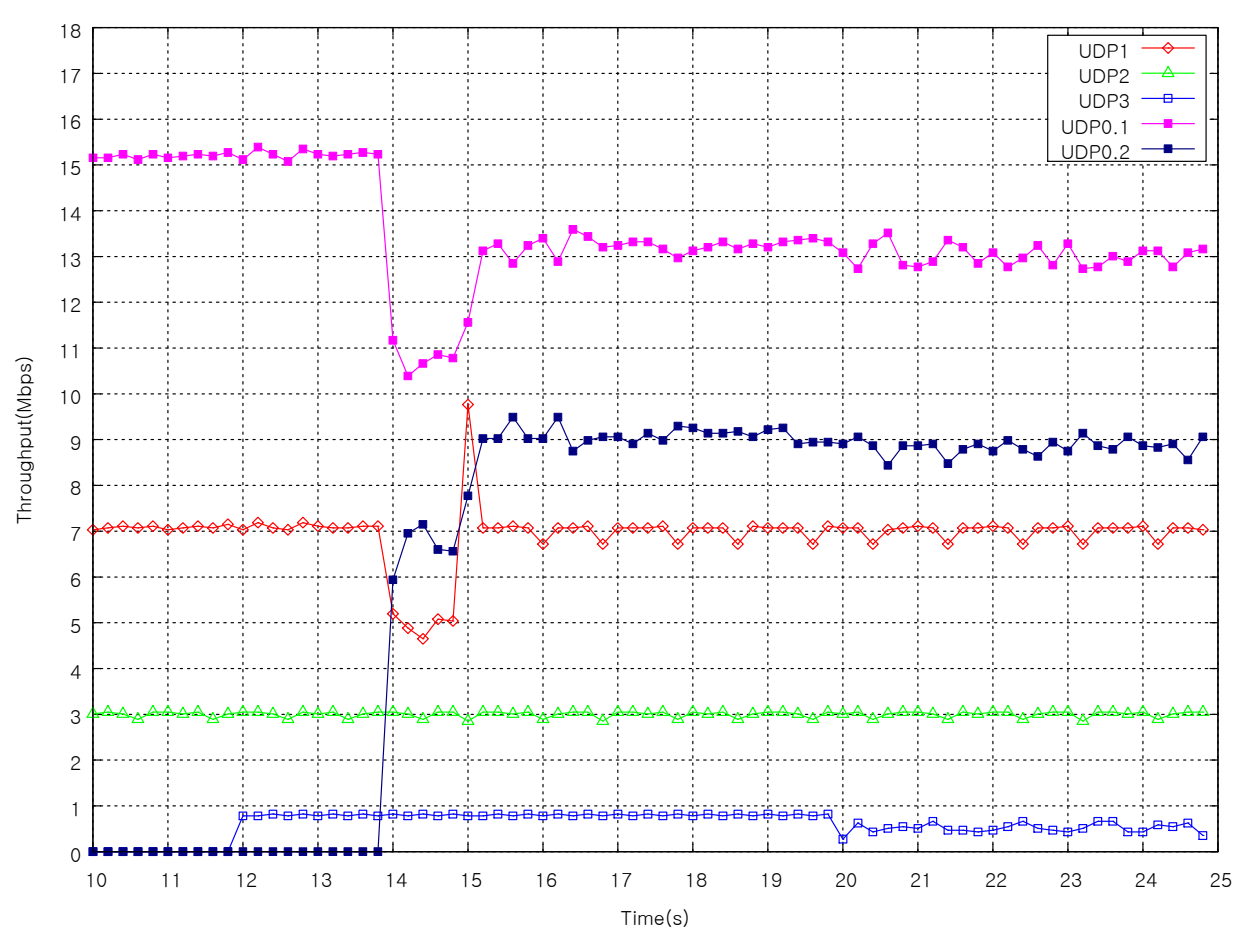

Fig. 10. UDP Throughput Trace of Phase 2 and 3 at the MN

UDP2 flow is. The reason of the rapid increase of throughput is that the MN receives UDP1 packets from both WLAN and WiMax interfaces simultaneously. It is also noted that there are oscillations in the throughput of the WiMax access network because WiMax transmit a series of packets at one time and it has an interval between transmissions.

In phase 3, MN-driven flow handover happens at 20.0 seconds, where the $\mathrm{MN}$ moves the UDP3 flow from the $3 \mathrm{G}$ interface to the WLAN interface. In the WLAN interface, there are two flows, UDP1 and UDP2 and thus the throughput of the UDP3 flow slightly decreases after the flow handover.

\section{CONCLUSION}

PMIPv6 is a network-based mobility support protocol and it does not require MNs to be involved in the mobility support signaling. Even though the MN's logical interface can solve the well-known problems of flow mobility in a heterogeneous network, the definite procedure for flow mobility has not been provided yet, and some missing procedures, such as $\mathrm{MN}$-derived flow handover, make PMIPv6-based flow mobility incomplete.

In this paper, an enhanced flow mobility support with $\mathrm{MN}$ logical interface has been presented for actualizing and fully covering the flow mobility support in PMIPv6. Enhanced flow mobility support is based on the logical interface in the MN. Logical interface hides all of the physical interfaces from the network layer and above. The Flow Interface Manager is placed at the 
logical interface and the Flow Binding Manager in the LMA is paired with it. They manage the flow bindings, and are used to select proper access technology to send the packet. Three different flow mobility triggering cases are defined, which are caused by new connections from the MN, the LMA's decision, and the MN's decision. Ns-3 simulation showed that the enhanced flow mobility support could perform well for possible flow mobility scenarios, including the MN-derived flow mobility support.

We have focused on the multi-homed MN for flow mobility support in this paper. As for our future work, we plan to perform an intensive performance evaluation of flow mobility, while including various handover scenarios with the mixture cases of multi-homed network handover, hard-handover, and soft-handover. Recently, the distributed mobility management has been studied a lot in order to overcome the limitations of the traditional centralized mobility management by bringing the mobility anchors closer to the MNs. We consider supporting a new flow mobility scheme under distributed mobility architecture.

\section{REFERENCES}

[1] S. Gundavelli, K. Leung, V. Devarapalli, K. Chowdhury and B. Patil, "Proxy Mobile IPv6," IETF RFC 5213, August, 2008.

[2] D. B. Johnson and C. E. Perkins and J. Arkko, "Mobility Support in IPv6," IETF RFC 3775, June, 2004.

[3] H. Soliman (Ed.), "Mobile IPv6 Support for Dual Stack Hosts and Routers," IETF RFC 5555, June, 2009.

[4] K. Kong, W. Lee, Y.-H. Han, M.-K. Shin and H.-R. You, "Mobility Management for All-lP Mobile Networks: Mobile IPv6 vs. Proxy Mobile IPv6," IEEE Wireless Communications, Vol.15, No.2, April, 2008, pp.36-45.

[5] M. Hui, G. Chen and H. Deng, "Service Flow Identifier in Proxy Mobile IPv6," draft-hui-netextservice-flow-identifier-03, July, 2010.

[6] F. Xia and B. Sarikaya, "Flow Binding in Proxy Mobile IPv6," draft-xia-netext-flow-binding-02, June, 2010.

[7] R. Koodli and K. Chowdhury, "Flow Handover for Proxy Mobile IPv6," draft-koodli-netext-flowhandover-01, October, 2009.

[8] S. Krishnan, H. Yokota, T. Melia and C. Bernardos, "Issues with Network based Inter-Technology Handovers," draft-krishnan-netext-intertech-ps-02, July, 2009.

[9] G. Tsirtsis, "Traffic Selectors for Flow Bindings," draft-ietf-mext-binary-ts-04, February, 2010.

[10] H. Yokota, S. Gundavelli, T. Tran, Y. Hong and K. Leung, "Virtual Interface Support for IP Hosts," draft-yokota-netlmm-pmipv6-mn-itho-support-03, March, 2010.

[11] T. Melia, Ed., "Logical Interface Support for multi-mode IP Hosts," draft-melia-netext-logialinterface-support-01, July, 2010.

[12] Y.-G. Hong, J.-S. Youn, H.-J. Kim, and T. Hyun, "Analysis of the Usage of a Logical Interface in PMIPv6," 13th International Conference on Advanced Communication Technology (ICACT), February, 2011, pp.1069-1074.

[13] CJ. Bernardos, Ed., "Proxy Mobile IPv6 Extensions to Support Flow Mobility," draft-bernardosnetext-pmipv6-flowmob-02, Feburary, 2011.

[14] T. Tran, Y. Hong, and Y. Han, "Flow Mobility Support in PMIPv6," draft-trung-netext-flow-mobilitysupport-01, October, 2010.

[15] H. Choi, S.-G. Min, Y.-H. Han, J. Park and H. Kim, "Implementation and Evaluation of Proxy Mobile IPv6 in NS-3 Network Simulator," In Proceedings of the IEEE International Conference on Ubiquitous Information Technology and Applications (CUTE), December, 2010, pp.1-6.

[16] H. Choi, S.-G. Min and Y.-H. Han, "PMIPv6-based Flow Mobility Simulation in NS-3," In Proceed- 
ings of International Conference on Innovative Mobile and Internet Services in Ubiquitous Computing (Workshop on Mobility Modeling and Performance Evaluation (MoMoPE)), December, 2010, pp.475-480.

[17] NS-3 Network Simulator, http://www.nsnam.org

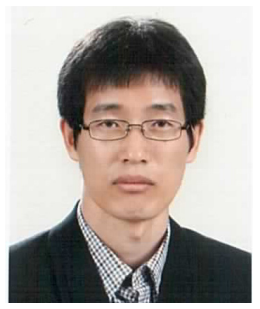

\section{Hyon-Young Choi}

He received B.S., M.S., Ph.D degrees in Computer Science and Engineering from Korea University, Seoul, Korea in 2003, 2005, and 2011, respectively. He has been a research professor in School of Convergence IT at Korea University, Seoul, Korea since 1 May 2011. His interests in research include QoS, mobility protocol design, and performance analysis.

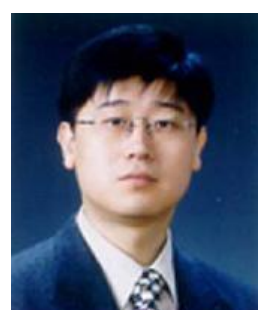

\section{Sung-Gi Min}

He received his B.S. degree in Computer Science from Korea University, Seoul, Korea, in 1988. He received his M.S. and Ph.D. degrees in Computer Science from University of London in 1989 and 1993, respectively. From 1 January 1994 to 28 February 2000, he worked in LG Information and Communication Research Center, and from 2 March 2000 to 28 February 2001, he was a Professor in the Department of Computer Engineering at Dongeui University, Busan, Korea. Since 2 March 2001, he has been a Professor in the Department of Computer Science and Engineering at Korea University, Seoul, Korea. His research is focused to wired/wireless communication networks, especially heterogeneous network environment, and he is interested in mobility protocols such as MIP, SIP, and SCTP, network architectures, QoS, and mobility management in future network.

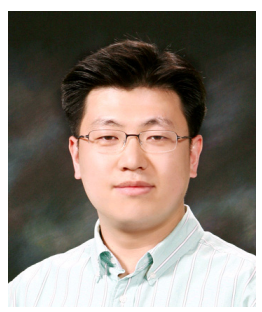

\section{Youn-Hee Han}

He received his B.S. degree in Mathematics from Korea University, Seoul, Korea, in 1996. He received his M.S. and Ph.D. degrees in Computer Science and Engineering from Korea University in 1998 and 2002, respectively. From 4 March 2002 to 28 February 2006, he was a senior researcher in the Next Generation Network Group of Samsung Advanced Institute of Technology. Since 2 March 2006, he has been a Professor in the School of Internet-Media Engineering at Korea University of Technology and Education, CheonAn, Korea. His primary research interests include theory and application of mobile computing, including protocol design and performance analysis. Since 2002, his activities have focused on Internet host mobility, sensor mobility, media independent handover, and cross-layer optimization for efficient mobility support on IEEE 802/LTE wireless networks. He has also made several contributions in IETF and IEEE standardization, and served as the chair of 'IPv6 over WiBro' working group in Korea TTA IPv6 Project Group. 


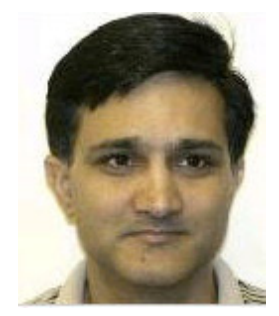

\section{Rajeev Koodli}

He received his BS degree in Electronics and Communication Engineering from Karnataka University, India, and his M.S. and Ph.D degrees in Computer Systems Engineering from the University of Massachusetts, Amherst, USA in 1993 and 1997 respectively. Since his graduation, he worked at Nokia Research Center as Senior Principal Scientist where he designed and contributed to the original IPv6 Mobility protocols including Mobile IPv6, Fast Handovers and Context Transfers. Rajeev joined Starent Networks as an Architect in 2008, where he contributed to the design of 4G LTE Core Network protocol standardization in 3GPP, including GTPV2, Proxy Mobile IPv6 and Diameter-based applications for 4G networks. In 2010, via the acquisition of Starent Networks, Rajeev joined Cisco Systems where he is currently a Principal Engineer and a Manager. His work involves Service Provider Network Virtualization, Mobility and Machine - Machine Communication. He has published fifteen IETF RFC publications and over twenty articles in International journals and conferences. He has served as Chair of the Mobility Optimizations Research Group in the Internet Research Task Force (IRTF), and currently serves as a co-chair of the IETF's NetExt Working Group. 